Original Research Article

\title{
Psychopharmacological characterization of effects of Ferula asafoetida linn. formulation in mouse on a Y-maze, EPM, and open field apparatus
}

\author{
Ramanujam Narayanan*, Bindu Gilbert, Santoshini Ravichandran, K. Bhuvaneswari
}

Department of Pharmacology, PSG IMSR, Coimbatore, Tamil Nadu, India

Received: 07 July 2017

Revised: 19 July 2017

Accepted: 29 July 2017

\section{*Correspondence to:}

Dr. Ramanujam Narayanan, Email: raam191983@gmail.com

Copyright: (c) the author(s), publisher and licensee Medip Academy. This is an openaccess article distributed under the terms of the Creative Commons Attribution NonCommercial License, which permits unrestricted noncommercial use, distribution, and reproduction in any medium, provided the original work is properly cited.

\begin{abstract}
Background: Asafoetida is an herbal food supplement that has numerous peripheral pharmacological actions. Its central nervous system effects have not yet been completely elucidated in both animals and human participants. The study focuses on deciphering the effects of this Herbaceutical with that of Donepezil and vitamin $\mathrm{C}$ in mouse models of short term novelty object recognition memory and open field test.
\end{abstract}

Methods: The study was conducted in two stages - a pilot stage which assessed the most effective dose of asafoetida on cognition, followed by a post-pilot study phase wherein its effects at that dose were compared with those of Donepezil and vitamin C. Novelty Preference Test (NPT) on y-maze and Open field test on Open field box were used to evaluate the effects of the formulation on memory and motor activity in mice.

Results: A dose of 400mg/day of asafoetida orally was found to improve memory significantly in comparison to $200 \mathrm{mg} /$ day in mice. Asafoetida showed a greater potency in improving memory than Donepezil and vitamin C. After 11 days of daily treatment with Asafoetida commercial powder, more than fifty percent of the mice showed an increase in recognition index of $0.7-0.8$ as compared to 0.55 at baseline.

Conclusions: Asafoetida powder has nootropic efficacy in mouse model - this has to be explored mechanistically and pharmaceutically to enable the psychopharmacological characterization of the active principles and its pharmacological effects in the central nervous system.

Keywords: Asafoetida, Donepezil, Elevated plus maze, Memory, Novelty object recognition memory, Open field test, Vitamin C, Y-Maze

\section{INTRODUCTION}

Asafoetida is a commercial form of dried latex extracted from the tap root of Ferula asafoetida linn. Plant, that is used in some countries as food supplement and flavouring agent. Although it has been widely used in Chinese and Indian medicine systems for thousands of years, its pharmacological effects have yet to be completely elucidated.

Different mechanism for augmentation of cognitive deficits exist in different pathophysiological states and disease- for example, Donepezil is a cholinesterase inhibitor which improves memory even in severe cognitive impairment seen in dementia by increasing acetylcholine levels in the cortical regions of the brain. ${ }^{1}$ Other mechanisms have also been proposed, including antioxidant actions of vitamins and minerals on free radicals and glutamate excitotoxicity prevention. ${ }^{2} \mathrm{We}$ propose to evaluate for the first time, the mechanism of nootropic action of a commercial preparation of the oleoresin of Ferula spp., although few studies have addressed the research question in other ways. For example, studies on its amnesia reversal in galactose and sodium nitrate induced deficits in rats and assessment of its cognitive effects using passive avoidance tests have been published with positive evidence of its central 
actions, although it has widespread effects in both preclinical and clinical models peripherally.,

The most commonly evaluated active principle of this herb is ferulic acid. ${ }^{5}$ For example, it contains sesquiterpenes, coumarins and sulphur-rich components and phenolics including ferulic acids. ${ }^{6-8}$

The present study aims to evaluate the most efficacious dose of asafoetida's nootropic effect and then to compare its cognitive effects with two probe drugs acting by different mechanisms viz., vitamin $\mathrm{C}$ and donepezil. This has been accomplished using a two-phase study evaluation approach.

\section{METHODS}

\section{Animals}

Prior animal ethics committee approval was obtained from the Institutional Animal Ethics Committee (IAEC) for thirty Swiss albino mice. After procurement from breeder and quarantine, these thirty mice were housed in groups of six, after separating them into two divisions, the first randomly selected twelve mice for pilot stage and the remaining eighteen mice for the post pilot efficacy study. The first twelve mice were initially acclimatized in the laboratory and then randomly reallocated after one week into two cages for the pilot study execution.

\section{Drugs}

Commercially available asafoetida powder is obtained and dissolved in water and administered by oral gavage in mice daily till a specific criterion is met with. Donepezil tablets are ground and dissolved in water and administered by intraperitoneal syringes daily. Vitamin $\mathrm{C}$ is obtained as tablets and dissolved in aqueous solvent for oral gavage $(\mathrm{pH}=7.0)$. The specific efficacy criterion is that treatment is continued in all groups till at least $50 \%$ of one either group exhibits a 50\% improvement in baseline NOR index earlier.

\section{Drug treatment}

The study comprised of two phases - an initial pilot phase and a second efficacy phase. The objective of the pilot study phase was to ascertain the more effective of two doses of asafoetida for meeting a predefined criterion of cognitive improvement, along with the standard drug Donepezil. The objective of the next phase was to compare the cognitive improvement of asafoetida with two standard drugs acting by different mechanism, using surrogate behavioural markers.

The mice in the first cage were administered $200 \mathrm{mg} / \mathrm{kg} /$ day of a commercial preparation of asafoetida powder in solution by oral gavage, whilst the other cage mice were given the same formulation at a dose of $400 \mathrm{mg} / \mathrm{kg} / \mathrm{day}$. Each mouse was also co-administered
Donepezil $2 \mathrm{mg} / \mathrm{kg} /$ day intraperitoneal, till at least $50 \%$ of the rodents given either combination exhibited a $15-20 \%$ improvement in novelty recognition indices from baseline. At this cut-off, treatment was stopped in both groups and an interim analysis was performed to ascertain which of the two doses is more potent in improving novelty object recognition memory on a y-maze.

The efficacy study phase was executed using eighteen mice which, after acclimatization, were divided into three groups - first group received Asafoetida 400mg/kg/day, the second group mice were administered Donepezil 2 $\mathrm{mg} / \mathrm{kg} /$ day intraperitoneal, and the six mice of the third group were given vitamin $\mathrm{C} 125 \mathrm{mg} / \mathrm{kg} /$ day $(\mathrm{pH}=7.0)$ orally.

\section{The Y-Maze}

The y-maze novelty preference test using the elevated ymaze for mouse evaluates short term spatial novelty-object recognition (NOR) memory. The elevated y-maze consists of three arms in a horizontal plane that is elevated from the floor by 50 centimetres. The three equally long arms are placed at $120^{\circ}$ to each other, separated by a triangular central platform. Each arm measures about 100 centimetres. In length and is enclosed by walls whose height is about 50 centimetres. One of the arms has a provision for closure at the junction with the central platform, with a guillotine door - this is the Novel (N) arm. The other two arms which do not have such doors are the Start (S) and the Familiar (F) arms. The NOR index calculated from the number of arm entries of a single mouse (NAE) into each arm, with 3/4ths of its body inside that arm, is as follows:

$$
\begin{gathered}
N O R \operatorname{INDEX}(\%)=\frac{N A E(N)}{N A E(N)+N A E(F)} \times 100 \\
\text { Familiarization index }=\frac{N A E(F)}{N A E(S)} \geq 1.0
\end{gathered}
$$

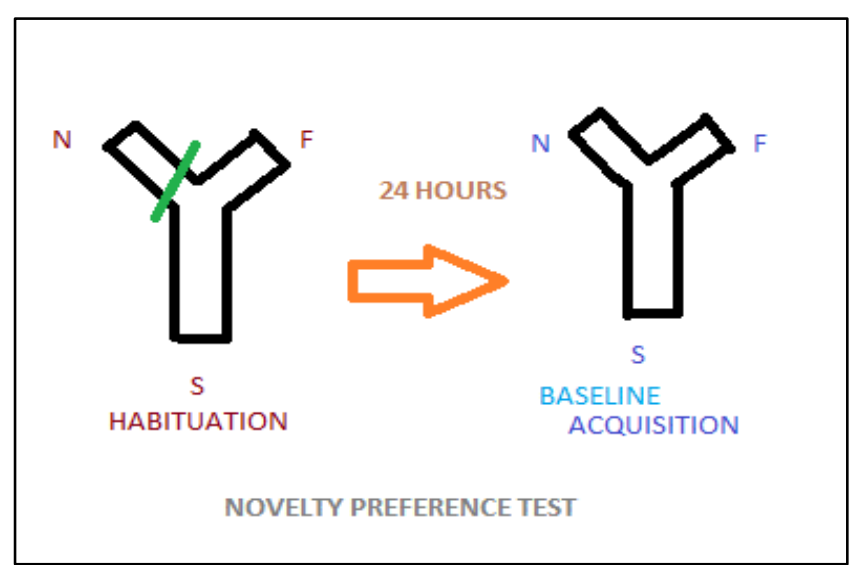

Figure 1: The Y Maze Novelty Preference test (NPT) protocol. The Novel arm $(\mathrm{N})$ is closed by the guillotine door (in green) in habituation but not in exploration and acquisition trials. 
The level of NOR memory acquisition and retention is directly proportional to the above index. The rodents are allowed to explore all three arms freely for 8 minutes in the y-maze followed by four successive habituation trials, in which the novel arm is closed. The habituation trials are meant for the rodent to familiarize with the familiar arm with novel arm closed. Thirty minutes after drug treatment, each animal can explore the novel arm relative to familiar arm, after placing it in the start arm for 5 minutes. NOR index is then measured for each animal (Figure 1).

\section{The open field test}

The open field box measures spontaneous motor activity (SMA) in rodents. ${ }^{4}$ It is a cubical box measuring $90 \mathrm{~cm}^{3}$ volume with an arena or floor, that is divided into 16 smaller yellow-bordered squares (Figure 2). The central portion of this arena is marked by a similar red-bordered square. The whole arena is divided into 4 white-bordered quadrants for purposes of software-acquisition of activity trials. The Open Field test measures SMA activity in 3minute trials per mouse. The SMA index as a measure of motor activity is calculated as follows:

$$
\text { SMA INDEX }=\frac{\sum \text { Peripheral yellow square entries }}{\sum \text { Central red square entry }}
$$

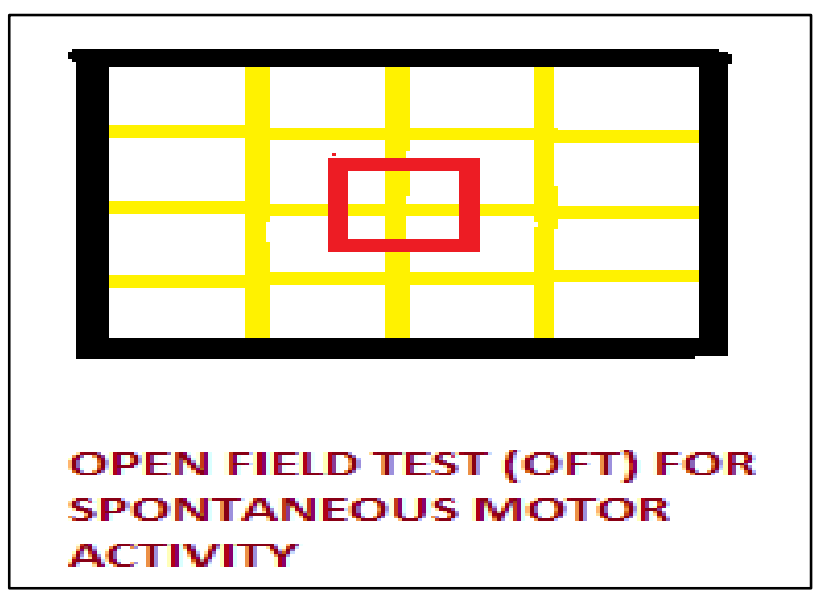

Figure 2: The open field box- note the peripheral squares in yellow and the central red square.

Elevated plus maze: The Elevated Plus Maze (EPM) is a plus like maze used to assess effects on interventions on anxiety like behaviour (Figure 3). It consists of four arms, two of which are enclosed on all three sides by walls. The other two arms which are on opposite end of the maze are not enclosed by walls. The whole maze is elevated on the ground at a height of $50 \mathrm{~cm}$. The transfer latency is the time the mouse takes to move from the open arm to the enclosed arm in a single daily ninety second trial. Additionally, the ratio of open to enclosed arm entries was also calculated as a measure of anxiolytic index.

The study design (Figure 4) is such that the more potent of two doses of asafoetida (with donepezil) is chosen based on greater / earlier nootropic activity followed by an efficacy phase, that aims to compare this dose of asafoetida with vitamin $\mathrm{C}$ and Donepezil-induced activities on $\mathrm{y}$ maze.

Statistical analysis: All statistical analysis was performed using Graphpad Prism v 4.0 and SPSS v 16.0. Analysis of variance ( 1 way and 2 way) with post hoc Bonferroni test and Pearson correlation along with Levenne test were performed for hypothesis testing.

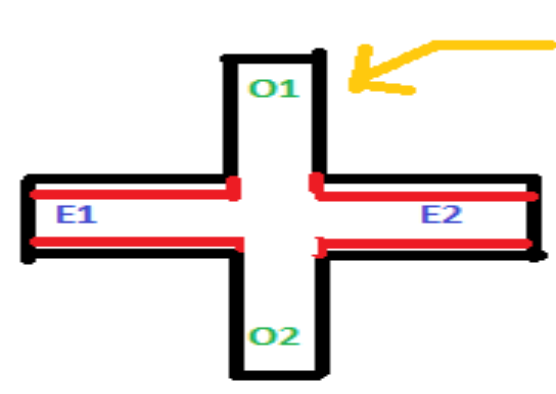

ELEVATED PLUS MAZE (EPM) TRANSFER LATENCY TEST

Figure 3: The elevated plus maze- the animal is placed on any one open arm end initially (shown in yellow arrow) to measure transfer latency.

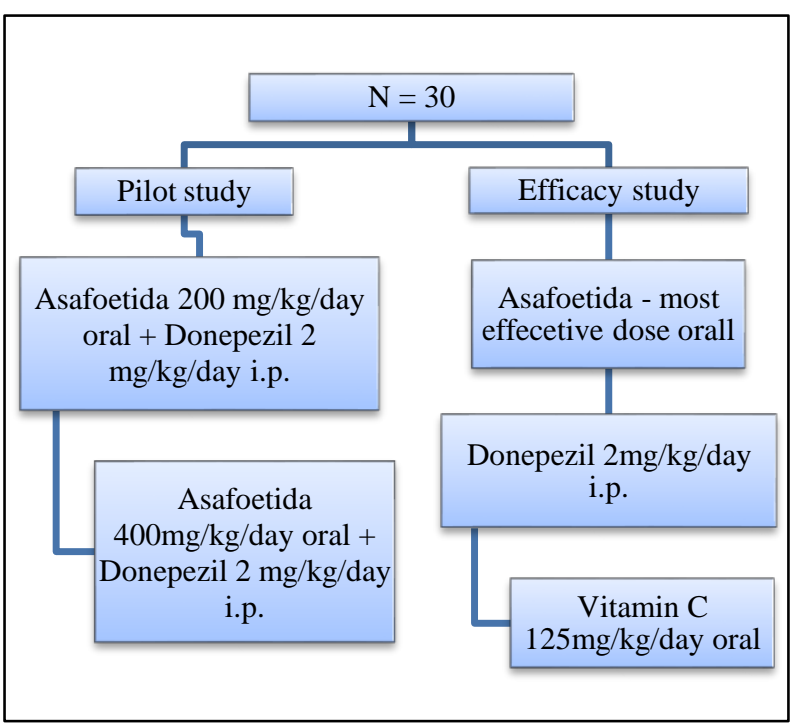

Figure 4: The study design for pilot and efficacy phases, with corresponding interventions.

\section{RESULTS}

There was a statistically significant difference between the groups as determined by one-way ANOVA after 7 days ( $F$ $(3,21)=3.581, \mathrm{p}=0.031)$ and after 14 days of drug treatment $(\mathrm{F}(3,21)=7.996, \mathrm{p}=0.001)$. A post hoc Bonferroni test with $\mathrm{Y}$ Maze Recognition index as the dependent variable across all groups of both phases taken together revealed a statistical significance between control 
and high dose asafoetida group $(\mathrm{p}=0.002)$. The improvement with high dose $(400 \mathrm{mg} / \mathrm{kg} /$ day) asafoetida was noted in 10 days of treatment even earlier compared to Donepezil in the efficacy study phase. After 11 days of daily treatment with Asafoetida commercial powder, more than fifty percent of the mice showed an increase in recognition index of $0.7-0.8$ as compared to 0.55 at baseline. A two-way ANOVA for determination of factors affecting the repeated measures of $\mathrm{Y}$ maze NOR Index revealed statistical significant for the influence of both days of exposure $(\mathrm{p}<0.0001)$ and drug treatment $(\mathrm{p}=$ 0.007). Pairwise group comparison across all four treatment groups revealed significant interaction of drug treatment over days - for example Asafoetida high dose was more nootropic than Donepezil on days 10 and 11, and vitamin $\mathrm{C}$ on day 11, in the Bonferroni test (Figure 5).

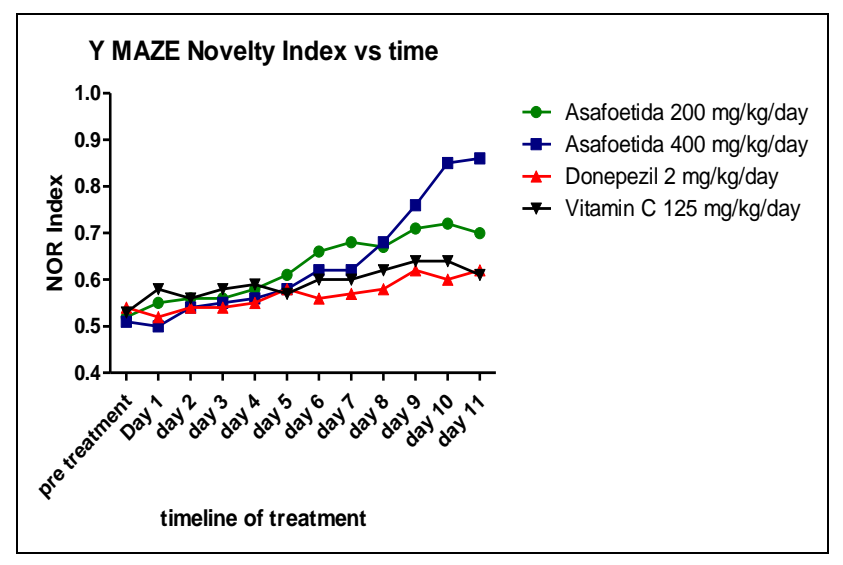

Figure 5: Mean NOR index on Y maze across treatment interventions versus time after baseline (pre-treatment) and treatment initiation (day 1) values.

The one-way ANOVA of open to enclosed arm entries on the EPM revealed statistical significance between Donepezil and high dose Asafoetida group ( $\mathrm{p}=0.017$, 95\% CI: -137.17 to -7.16 ), with the mean ratios greater in the asafoetida $400 \mathrm{mg} / \mathrm{kg} /$ day group ( $\mathrm{p}=0.024$ ), as revealed by a Bonferroni test. The percentage of open to enclosed arm entry ratios on days 7 and 14 across all the groups did not yield statistical significance using Levenne test $(\mathrm{p}>0.05)$.

Intergroup comparison of the ratio of peripheral to red square ratios on open field on days 7 and 14 across all the treated groups revealed no statistical significance using 1way ANOVA $(\mathrm{p}>0.05)$. Also, the number of red and peripheral square entries on open field test on days 7 and 14 did not yield significant differences among all groups.

The Pearson correlation coefficient between y maze recognition index and the ratio of peripheral to central square entries on open field showed significant correlations on day $7\left(\mathrm{R}^{2}=0.8\right)$, but not on day 14 , and both the correlations were statistically insignificant. A similar result was obtained for correlation between recognition index and transfer latency.

\section{DISCUSSION}

A novel effect of Asafoetida on spatial memory on y-maze which was earlier and greater than even Donepezil has been reported in this study. The mechanism of cognitive improvement seems to correlate more with cholinergic pathway improvement with Donepezil than with vitamin $\mathrm{C}$ effects. Also, this nootropic effect of Donepezil was higher at high dose with minimal motor and anxiogenic side effects as noted by results of open field and elevated plus maze tests.

Vitamin C exerts neuroprotective effects after its transport into neurons through sodium-dependent ascorbate transporters. ${ }^{1}$ Donepezil is a cerebroselective acetylcholinesterase inhibitor that increases neurogenesis in the central nervous system, especially the hippocampus, and improves cognition. ${ }^{2}$

Although only a couple of studies exist to prove asafoetida nootropic effects, ${ }^{3,4}$ our study reports that it affects spatial memory with minimal motor and anxiety adverse effects, which parallels more with Donepezil mechanism than antioxidant pathway, from the recognition indices parallelism.

Derivatives of sesquiterpenes are known to have chemo preventive effects in cancers and coumarins have anticoagulant activity. ${ }^{5-7}$ Ferulic acid have also been used to improve haemostatics during silk weaving. ${ }^{8,9}$ Phenolic acids which includes about half a dozen chemicals in Ferula spp. possess emotional improving and antidepressant activities. However, its effects in the central nervous system on higher cognitive properties have not been deciphered and evaluated completely.

\section{Limitations}

A small sample size and absence of biochemical confirmation; but it will serve as a pilot study for enabling further studies on this important nutraceutical.

Funding: No funding sources Conflict of interest: None declared

Ethical approval: The study was approved by the Institutional Animal Ethics Committee

\section{REFERENCES}

1. Hansen SN, Tveden-Nyborg P, Lykkesfeldt J. Does Vitamin C Deficiency Affect Cognitive Development and Function? Nutrients. 2014;6(9):3818-46.

2. Jacobsen FM, Comas-Díaz L. Donepezil for psychotropic-induced memory loss. J Clin Psychiatry. 1999 Oct;60(10):698-704.

3. Bagheri SM, Dashti-R MH. Influence of asafoetida on prevention and treatment of memory impairment 
induced by d-galactose and $\mathrm{NaNO} 2$ in mice. Am J Alzheimers Dis Other Demen. 2015 Sep;30(6):60712.

4. Vijayalakshmi, Adiga S, Bhat P, Chaturvedi A, Bairy KL, Kamath S. Evaluation of the effect of Ferula asafoetida Linn. gum extract on learning and memory in Wistar rats, Ind J Pharmacol. 2012;44(1):82-7.

5. Augustine A, Sreeraj G. Biological activities and medicinal properties of Asafoetida: A review, Journal of Traditional and Complementary Medicine; 2016. ISSN 2225-4110. Available at: http://dx.doi.org/10.1016/j.jtcme.2016.11.004.

6. Iranshahy M, Iranshahi M. Traditional uses, phytochemistry and pharmacology of asafoetida (Ferula assa-foetida oleo-gum-resin): A review. Journal of ethnopharmacology. 2011 Mar 8;134(1):10 .

7. Kogure K, Yamauchi I, Tokumura A, Kondou K, Tanaka N, Takaishi $\mathrm{Y}$, et al. Novel antioxidants isolated from plants of the genera Ferula, Inula,
Prangos and Rheum collected in Uzbekistan. Phytomedicine. 2004 Nov 25;11(7):645-51.

8. Singh UP, Singh DP, Maurya S, Maheshwari R, Singh M, Dubey RS, et al. Investigation on the phenolics of some spices having pharmacotherapeutic properties. Journal of herbal pharmacotherapy. 2004 Jan 1;4(4):27-42.

9. Wang S, Gao Z, Chen X, Lian X, Zhu H, Zheng J, et al. The anticoagulant ability of ferulic acid and its applications for improving the blood compatibility of silk fibroin. Biomedical Materials. 2008 Nov 25;3(4):044106.

Cite this article as: Ramanujam N, Bindu G,

Santoshini R, Bhuvaneswari K.

Psychopharmacological characterization of effects of Ferula asafoetida linn. formulation in mouse on a Ymaze, EPM, and open field apparatus. Int J Basic Clin Pharmacol 2017;6:2254-8. 